TRANSACTIONS OF THE

AMERICAN MATHEMATICAL SOCIETY

Volume 352 Number 4 , Pages 1623-1633

S 0002-9947(99)02302-8

Article electronically published on December 10, 1999

\title{
ON CHOW MOTIVES OF 3-FOLDS
}

\author{
PEDRO LUIS DEL ANGEL AND STEFAN MÜLLER-STACH
}

\begin{abstract}
Let $k$ be a field of characteristic zero. For every smooth, projective $k$-variety $Y$ of dimension $n$ which admits a connected, proper morphism $f$ : $Y \rightarrow S$ of relative dimension one, we construct idempotent correspondences (projectors) $\pi_{i j}(Y) \in C H^{n}(Y \times Y, \mathbb{Q})$ generalizing a construction of Murre. If $n=3$ and the transcendental cohomology group $H_{\mathrm{tr}}^{2}(Y)$ has the property that $H_{\mathrm{tr}}^{2}(Y, \mathbb{C})=f^{*} H_{\mathrm{tr}}^{2}(S, \mathbb{C})+\operatorname{Im}\left(f^{*} H^{1}(S, \mathbb{C}) \otimes H^{1}(Y, \mathbb{C}) \rightarrow H_{\mathrm{tr}}^{2}(Y, \mathbb{C})\right)$, then we can construct a projector $\pi_{2}(Y)$ which lifts the second Künneth component of the diagonal of $Y$. Using this we prove that many smooth projective 3 -folds $X$ over $k$ admit a Chow-Künneth decomposition $\Delta=p_{0}+\ldots+p_{6}$ of the diagonal in $C H^{3}(X \times X, \mathbb{Q})$.
\end{abstract}

\section{INTRODUCTION}

Definition. Let $X \in S m(k)$ be a smooth, projective $k$-variety of dimension $d$. We say that $X$ has a Chow-Künneth decomposition, if there exist projectors $p_{0}, p_{1}, \ldots, p_{2 d}$ in $C H^{d}(X \times X) \otimes \mathbb{Q}$ such that the following properties hold:

(1) $p_{j} \circ p_{i}=\delta_{i j} \cdot p_{i}$.

(2) $\Delta=\sum p_{i}$.

(3) In étale cohomology the $p_{i}$ induce the $(2 d-i, i)-$ th Künneth component of the diagonal.

We say that $X$ has a Murre decomposition, if additionally the following properties hold:

(4) $p_{0}, \ldots, p_{j-1}$ and $p_{2 j+1}, . ., p_{2 d}$ act trivially on $C H^{j}(X) \otimes \mathbb{Q}$.

(5) If we put $F^{0} C H^{j}(X) \otimes \mathbb{Q}=C H^{j}(X) \otimes \mathbb{Q}$ and inductively $F^{k} C H^{j}(X) \otimes \mathbb{Q}:=$ $\operatorname{Ker}\left(\left.p_{2 j+1-k}\right|_{F^{k-1}}\right)$, then this descending filtration is intrinsic.

(6) Always $F^{1} C H^{j}(X) \otimes \mathbb{Q}=C H_{h o m}^{j}(X) \otimes \mathbb{Q}$.

Finally a Chow-Künneth decomposition $\left\{p_{i}(X)\right\}$ of $X$ is called special, if the following properties are satisfied: $p_{\ell}(X)$ is supported on $V_{\ell} \times X$ for every $0 \leq \ell \leq$ $d-1$, where $V_{\ell} \subset X$ is a closed subset of dimension $\ell$ and $p_{d-\ell}=p_{d+\ell}^{\mathrm{tr}}$ for all $\ell$.

Conjecture (J. Murre). Every smooth projective variety has a Murre decomposition.

Parts of (1)-(6) have been proved for curves [K], [M], surfaces [Mu2], products of a curve and a surface [Mu1, uniruled 3-folds dAMS, elliptic modular 3-folds GM],

Received by the editors September 20, 1997.

1991 Mathematics Subject Classification. Primary 14C25, 14E10; Secondary 19E15.

Key words and phrases. Chow group, correspondence, motive, Albanese map.

The first author was supported in part by DFG and CONACYT.

The second author was supported in part by DFG. 
abelian varieties $[\mathrm{Sh},[\mathrm{B}],[\mathrm{DM}],[\mathrm{Kü}]$ certain moduli spaces $[\mathrm{dBR}]$ and generalized flag varieties $\underline{\mathrm{K}}$ ].

In this paper we want to generalize the results of [AMS], where certain correspondences were constructed in order to obtain a Chow-Künneth decomposition (see Mu1]) of a smooth uniruled projective 3 -fold $X$ over $\mathbb{C}$ :

Theorem 1 (dAMS). Let $X$ be a uniruled complex projective 3-fold. Then $X$ admits a special Chow-Künneth decomposition with properties (4) and (6) of a Murre decomposition.

This construction worked mainly, because - in the case where $X$ is birational to a conic bundle - a certain blow-up $Y$ of $X$ has the property that there is a morphism $f: Y \rightarrow S$ such that the general fiber is a smooth rational curve.

The main observation in this paper is that one can in certain cases allow that the generic fiber is of positive genus: let $X$ be a smooth, projective 3-fold over a field $k$ of characteristic zero. Let $\sigma: Y \rightarrow X$ be a blow-up and assume that there is a connected proper morphism $f: Y \rightarrow S$, generically smooth of relative dimension one onto a smooth surface $S$. Let $i: Z \hookrightarrow Y$ be a smooth divisor finite over $S$. Then there exist projectors (=idempotent correspondences) in $Y \times Y$,

$$
\begin{aligned}
\pi_{i 0} & :=\frac{1}{m}(i \times 1)_{*}(h \times f)^{*} \pi_{i}(S), \\
\pi_{i 2} & :=\frac{1}{m}(1 \times i)_{*}(f \times h)^{*} \pi_{i}(S), \\
\pi_{i 1} & :=\frac{1}{\operatorname{deg}(\epsilon)}(f \times f)^{*}\left(\pi_{i}(S)\right) \cdot D .
\end{aligned}
$$

for $0 \leq i \leq 4$ which can be combined to form a Chow-Künneth decomposition of $Y$ (and therefore later on $X$ ) if the following condition is satisfied:

Definition. Let $f: Y \rightarrow S$ be as above. We say that $f$ decomposes $H_{\mathrm{tr}}^{2}(Y)$, if $H_{\mathrm{tr}}^{2}(Y)=f^{*} H_{\mathrm{tr}}^{2}(S, \mathbb{C})+\operatorname{Im}\left(f^{*} H^{1}(S, \mathbb{C}) \otimes H^{1}(Y, \mathbb{C}) \rightarrow H_{\mathrm{tr}}^{2}(Y)\right)$.

Here we defined $H_{\mathrm{tr}}^{2}(Y):=H^{2}(Y, \mathbb{C}) / N S(Y) \otimes \mathbb{C}$. This notion is a generalization of isotriviality and means that the cohomology classes in $H_{\mathrm{tr}}^{2}(Y, \mathbb{C})$ behave somewhat like the situation where $Y$ is the product of $S$ with some curve. In this paper we therefore prove the following generalization of theorem 1:

Theorem 2. Let $X$ be a smooth projective 3-fold over a field of characteristic 0 such that a blow-up $Y$ of $X$ has a fibration $f: Y \rightarrow S$ which decomposes $H_{t r}^{2}(Y)$. Then $X$ admits a special Chow-Künneth decomposition.

Examples. Let $S$ be a smooth projective surface. Consider a smooth ample hypersurface $Y$ in $S \times S_{1}$, where $S_{1}$ is a smooth, projective surface with $H_{\mathrm{tr}}^{2}\left(S_{1}\right)=0$. Let $f=p r_{1}$ be the first projection. By the Lefschetz theorems, $f=p r_{1}: Y \rightarrow S$ decomposes $H_{\mathrm{tr}}^{2}(Y)$. Cyclic branched coverings of $S \times C$ (C a smooth curve) also have this property.

\section{ACKNOWLEDGEMENTS}

We are thankful to H. Esnault, B. Gordon, D. Huybrechts, J. Nagel and E. Viehweg for helpful discussions and several improvements of our exposition. J. Murre has pointed out a gap in a previous version and we are especially thankful to him. 


\section{NEW CORRESPONDENCES}

In this section we generalize the construction of J. Murre from Mu2]. Let $S$ be an $n$-1-dimensional smooth projective variety and assume that there exist a Chow-Künneth decomposition for $S$ given by projectors $\pi_{0}(S), \ldots, \pi_{2 n-2}(S)$ satisfying $\pi_{i}(S)^{\operatorname{tr}}=\pi_{2 n-2-i}(S)$. We assume that $f: Y \longrightarrow S$ is a proper, connected morphism generically smooth of relative dimension one. Let $Z \subset Y$ be a smooth divisor and assume that the morphism $h=\left.f\right|_{Z}: Z \longrightarrow S$ has finite fibers.

From now on suppose that the ground field $k$ is big enough such that $Z$ and $f$ are defined over $k$. This is not a serious restriction by [Sch, 1.17]. Let $e$ be a $k$-rational point in $S, F=f^{-1}(e), p$ a $k$-rational point in $F \cap Z$ and $m=(Z \cdot F)$. There are natural morphisms

$$
\operatorname{Pic}^{0}(Y) \longrightarrow P i c^{0}(F) \cong A l b(F) \longrightarrow A l b(Y)
$$

which do not induce, in general, an isogeny. Nevertheless, if one takes $K=$ $\operatorname{ker}(\operatorname{Alb}(Y) \longrightarrow \operatorname{Alb}(S))$, this maps does induce an isogeny $\epsilon: K^{\vee} \rightarrow K$ which has a factorization

$$
\epsilon: K^{\vee}=\operatorname{Pic}^{0}(Y) / f^{*} \operatorname{Pic}^{0}(S) \rightarrow \operatorname{Pic}^{0}(F) \cong A l b(F) \rightarrow K .
$$

Here the point is that the restriction map $H^{1}(Y) / f^{*} H^{1}(S) \rightarrow H^{1}(F)$ is injective in our situation. This can be seen as follows: Let $\Sigma \subset S$ be the non-smooth locus of $f$ and $T:=f^{-1}(\Sigma)$. Using the localization sequence on $Y$ and $S$ we obtain a commutative diagram

$$
\begin{aligned}
& 0 \rightarrow H^{1}(S) \quad \rightarrow \quad H^{1}(S \backslash \Sigma) \rightarrow H_{\Sigma}^{2}(S) \\
& \downarrow f^{*} \quad \downarrow f^{*} \quad \cap \\
& 0 \rightarrow H^{1}(Y) \quad \rightarrow \quad H^{1}(Y \backslash T) \quad \rightarrow \quad H_{T}^{2}(Y)
\end{aligned}
$$

which, together with the degeneration of the Leray spectral sequence over $S \backslash \Sigma$, shows that $H^{1}(Y) / f^{*} H^{1}(S) \subset H^{0}\left(S \backslash \Sigma, R^{1} f_{*} \mathbb{C}\right) \subset H^{1}(F)$ maps isomorphically to the invariant part of $H^{1}(F)$.

One has an inverse isogeny $\epsilon^{\text {tr }}: K \rightarrow K^{\vee}$. Now we can define a map $Y \rightarrow K$ by composing the Albanese map alb $: Y \rightarrow A l b(Y)$ with base point $p$ with the projection $\operatorname{Alb}(Y) \rightarrow K$, which is induced by the multisection $Z \subset Y$. Thus we obtain a map

$$
Y \times Y \rightarrow K \times K^{i d \times \epsilon^{\mathrm{tr}}} \mathrm{\longrightarrow} \times K^{\vee}
$$

and we let $D$ be the pullback of the normalized Poincaré divisor. This divisor $D$ will play an essential role in what follows and has the following properties:

$$
(*): D \cap\{p\} \times Y=Y \times\{p\} \cap D=0, \quad(f \times 1)_{*} D=0 .
$$

The last property follows from the fact that the degree of $D$ over $S \times Y$ is zero by definition of $D$.

Lemma 1. With the notation above, one has

$$
(1 \times f \times 1)_{*}(D \times Y \cap Y \times D)=\operatorname{deg}(\epsilon)\left(\operatorname{pr}_{13}^{Y \times S \times Y}\right)^{*} D .
$$

Proof. By the theorem of the cube it is enough to show that the left hand side and the right hand side coincide when restricted to $Y \times S \times\{p\}, Y \times\{e\} \times Y$ and $\{p\} \times S \times Y$. 
For the right hand side one has

$$
\left(p r_{13}^{Y \times S \times Y}\right)^{*} D \cap\{p\} \times S \times Y=\left(p r_{13}^{Y \times S \times Y}\right)^{*}(D \cap\{p\} \times Y)=0,
$$

while for the left hand side one gets

$$
\begin{aligned}
& (1 \times f \times 1)_{*}(D \times Y \cap Y \times D) \cap\{p\} \times S \times Y \\
& \quad=(1 \times f \times 1)_{*}[(D \cap\{p\} \times Y) \times Y \cap Y \times D]=0
\end{aligned}
$$

by $(*)$. In a similar fashion

$$
\left(p r_{13}^{Y \times S \times Y}\right)^{*} D \cap Y \times S \times\{p\}=\left(p r_{13}^{Y \times S \times Y}\right)^{*}(D \cap Y \times\{p\})=0
$$

and

$$
\begin{aligned}
& (1 \times f \times 1)_{*}(D \times Y \cap Y \times D) \cap Y \times S \times\{p\} \\
& \quad=(1 \times f \times 1)_{*}[Y \times(D \cap Y \times\{p\}) \cap D \times Y]=0
\end{aligned}
$$

by $(*)$. Finally the right hand side restricted to $Y \times\{e\} \times Y$ is simply $\operatorname{deg}(\epsilon) \cdot D$ whereas the left hand side we abbreviate by $E$ for a moment. Observe that $E$ also is a pull back from $K \times K$, by the following base change diagram (using [Fu, 1.7])

$$
\begin{array}{cccc}
Y \times F \times Y & \rightarrow & K \times F \times K & \hookrightarrow \\
\downarrow & & \downarrow & \\
Y \times\{e\} \times Y & \rightarrow & K \times\{e\} \times K &
\end{array}
$$

By the universal property of Poincaré divisors (see Mum) it is sufficient to check that $E$ acts as a correspondence $E: K \rightarrow K^{\vee}$ in the same way as $\operatorname{deg}(\epsilon) \cdot D$ does. Let $W$ be a zero cycle such that its Albanese image is in $K$. Then, by a computation in the following diagram

$$
\begin{array}{ccc}
Y \times F \times Y & \stackrel{1 \times i \times 1}{\longrightarrow} & Y \times Y \times Y \\
1 \times f \times 1 \downarrow & \downarrow p r_{3} \\
Y \times\{e\} \times Y & \stackrel{p r_{3}}{\longrightarrow} & Y
\end{array}
$$

we finally compute

$$
\begin{gathered}
E(W)=p r_{2 *}^{Y \times Y}(W \times Y \cap E) \\
=p r_{3 *}^{Y \times Y \times Y}[D \times Y \cap Y \times D \cap W \times Y \times Y \cap Y \times F \times Y] \\
=p r_{2 *}^{Y \times Y} p r_{23 *}^{Y \times Y \times Y}[D \times Y \cap W \times Y \times Y \cap Y \times D \cap Y \times F \times Y] \\
=p r_{2 *}^{Y \times Y}\left[\epsilon^{\operatorname{tr}}(W) \times Y \cap F \times Y \cap D\right] \\
=p r_{2 *}^{Y \times Y}[\operatorname{deg}(\epsilon) \cdot W \times Y \cap D]=\operatorname{deg}(\epsilon) D(W) .
\end{gathered}
$$

In the above computation we used the following fact: the composition of the following maps and correspondences equals $\epsilon \circ \epsilon^{t r}=\operatorname{deg}(\epsilon) \cdot I d$ :

$$
K \hookrightarrow A l b(Y) \stackrel{D}{\rightarrow} \operatorname{Pic}^{0}(Y) \rightarrow K^{\vee} \rightarrow \operatorname{Pic}^{0}(F) \cong A l b(F) \rightarrow K .
$$

Hence $E$ and $\operatorname{deg}(\epsilon) \cdot D$ act identically on $K$. That proves the assertion. 
For $j=0,1,2$ and $0 \leq i \leq 2 n-2$ one defines a cycle $\pi_{i j}$ as follows:

$$
\begin{aligned}
\pi_{i 0} & :=\frac{1}{m}(i \times 1)_{*}(h \times f)^{*} \pi_{i}(S), \\
\pi_{i 2} & :=\frac{1}{m}(1 \times i)_{*}(f \times h)^{*} \pi_{i}(S), \\
\pi_{i 1} & :=\frac{1}{\operatorname{deg}(\epsilon)}(f \times f)^{*}\left(\pi_{i}(S)\right) \cdot D .
\end{aligned}
$$

Proposition 1. The $\pi_{i j}$ satisfy the following properties:

(1) $\pi_{i k} \circ \pi_{j k}=\delta_{i j} \pi_{i k}$, in particular all $\pi_{i k}$ are projectors.

(2) $\pi_{i 1} \circ \pi_{j 0}=0$.

(3) $\pi_{i 2} \circ \pi_{j 1}=0$.

(4) $\pi_{i 2} \circ \pi_{j 0}=0$.

Proof. If no $\pi_{i 1}$ is involved, the result follows easily as in dAMS. On the other hand

$$
\begin{aligned}
& \operatorname{deg}(\epsilon)^{2}\left(\pi_{i 1} \circ \pi_{j 1}\right) \\
& =\left(p r_{13}^{Y \times Y \times Y}\right)_{*}\left[(f \times f \times f)^{*}\left(\pi_{j}(S) \times S \cap S \times \pi_{i}(S)\right) \cap D \times Y \cap Y \times D\right] \\
& =\left(p r_{13}\right)_{*}\left[(f \times 1 \times f)^{*}\left(\pi_{j}(S) \times S \cap S \times \pi_{i}(S)\right) \cap(1 \times f \times 1)_{*}(D \times Y \cap Y \times D)\right] \\
& =\operatorname{deg}(\epsilon)^{2} \cdot \delta_{i j} \pi_{i 1}
\end{aligned}
$$

since $(1 \times f \times 1)_{*}(D \times Y \cap Y \times D)=\operatorname{deg}(\epsilon)\left(p r_{13}^{Y \times S \times Y}\right)^{*} D$ by lemma 1 .

For $\pi_{i 1} \circ \pi_{j 0}\left(\right.$ or $\left.\pi_{j 2} \circ \pi_{i 1}\right)$, one needs that $(f \times 1)_{*} D=0$ by $(*)$.

Proposition 2. $\pi_{02}, \pi_{11}, \pi_{20}$ satisfy the following additional properties:

(1) $\pi_{20}=i d$ on $f^{*} H^{2}(S, \mathbb{Q})$.

(2) $\pi_{02}=i d$ on $\mathbb{Q} \cdot[Z]$ and $\pi_{02}=0$ on $f^{*} H_{t r}^{2}(S, \mathbb{Q})$.

(3) $\pi_{11}=0$ on $f^{*} H^{2}(S, \mathbb{Q})$ and $\pi_{11}=i d$ on

$$
\operatorname{Im}\left(f^{*} H^{1}(S, \mathbb{Q}) \otimes H^{1}(Y, \mathbb{Q}) \rightarrow H_{t r}^{2}(Y, \mathbb{Q}) / \operatorname{Imf}^{*} H^{2}(S, \mathbb{Q})\right) .
$$

(4) If furthermore $f: Y \rightarrow S$ decomposes $H_{t r}^{2}(Y)$, then $\pi_{2}:=\pi_{20}+\pi_{02}+\pi_{11}-$ $\pi_{20} \pi_{02}-\pi_{11} \pi_{02}-\pi_{20} \pi_{11}+\pi_{20} \pi_{11} \pi_{02}$ is a projector that acts as the identity on $H_{t r}^{2}(Y, \mathbb{Q})$.

Proof. (1) and (2) are proven in dAMS. (3): On $f^{*} H^{2}(S, \mathbb{Q})$ we have by $(1)$ that $\pi_{20}=i d$ therefore $\pi_{11}=\pi_{11} \circ \pi_{20}=0$ on $f^{*} H^{2}(S, \mathbb{Q})$ by proposition 1 . To prove the rest of (3) we have to make an explicit computation. Let

$$
f^{*} \alpha \wedge \beta \in \operatorname{Im}\left(f^{*} H^{1}(S, \mathbb{Q}) \otimes H^{1}(Y, \mathbb{Q}) \rightarrow H_{\mathrm{tr}}^{2}(Y, \mathbb{Q})\right)
$$

be representing differential forms. Then (as currents)

$$
\begin{gathered}
\pi_{11}\left(f^{*} \alpha \wedge \beta\right)=\frac{1}{\operatorname{deg}(\epsilon)} p r_{2 *}^{Y \times Y}\left[(f \times f)^{*} \pi_{1}(S) \cap D \cap\left(f^{*} \alpha \wedge \beta\right) \times Y\right] \\
=\frac{1}{\operatorname{deg}(\epsilon)} p r_{2 *}^{S \times Y}\left[(1 \times f)^{*}\left(\pi_{1}(S) \cap \alpha \times S\right) \cap(f \times 1)_{*}(D \cap \beta \times Y)\right]
\end{gathered}
$$


by the projection formula. Now let $\gamma:=(f \times 1)_{*}(D \cap \beta \times Y)$, a 1-current on $S \times Y$. By the Künneth formula, we obtain $\gamma=p r_{1}^{*}\left(\gamma_{1}\right)+p r_{2}^{*}\left(\gamma_{2}\right)$. Hence we get

$$
\begin{aligned}
\pi_{11}\left(f^{*} \alpha \wedge \beta\right)= & \frac{1}{\operatorname{deg}(\epsilon)} p r_{2 *}^{S \times Y}\left[(1 \times f)^{*}\left(\pi_{1}(S) \cap\left(\alpha \wedge \gamma_{1}\right) \times Y\right)\right] \\
& +\frac{1}{\operatorname{deg}(\epsilon)} p r_{2 *}^{S \times Y}\left[(1 \times f)^{*}\left(\pi_{1}(S) \cap \alpha \times Y\right)\right] \wedge \gamma_{2} \\
= & \frac{1}{\operatorname{deg}(\epsilon)}\left[f^{*} \pi_{1}(S)\left(\alpha \wedge \gamma_{1}\right)+f^{*} \alpha \wedge \gamma_{2}\right]
\end{aligned}
$$

since $\pi_{1}(S)$ is the identity on $H^{1}(S)$.

But the first term is zero, since $\pi_{1}(S)$ acts trivially on $H^{2}(S)$. Therefore we have shown that $\pi_{11}\left(f^{*} \alpha \wedge \beta\right)=\frac{1}{\operatorname{deg}(\epsilon)} f^{*} \alpha \wedge \gamma_{2}$. It remains to prove that

$$
\beta-\frac{1}{\operatorname{deg}(\epsilon)} \gamma_{2} \in f^{*} H^{1}(S, \mathbb{Q}) \text {. }
$$

Recall that $H^{1}(Y) / f^{*} H^{1}(S) \hookrightarrow H^{1}(F)$ is an injection. Thus we have only to show that after restriction to $F \times F$ one has

$$
\frac{1}{\operatorname{deg}(\epsilon)}(f \times 1)_{*}\left(\left.D\right|_{F \times F} \cap \delta \times F\right)=\delta
$$

for all $\delta \in H^{1}(K) \subset H^{1}(F, \mathbb{Q})$. However on $F \times F$ the map $(f \times 1)_{*}$ equals $p r_{2 *}$ and the assertion follows from the fact that $\frac{1}{\operatorname{deg}(\epsilon)} p r_{2 *}\left(\left.D\right|_{F \times F} \cap p r_{1}^{*} \delta\right)=\delta$, which in turn is a consequence of the fact that the projector $\left.\frac{1}{\operatorname{deg}(\epsilon)} D\right|_{F \times F} \in C H^{1}(F \times F, \mathbb{Q})$ induces by construction the projection map $H^{1}(F) \rightarrow H^{1}(K) \subset H^{1}(F)$. This proves (3).

Finally (4) is a formal consequence of (1)-(3). If $a, b, c$ are elements of a ring $R$ with $a^{2}=a, b^{2}=b, c^{2}=c, b a=c a=c b=0$ and we set $p=a+b+c-a b-$ $a c-b c+a b c$, then $p^{2}=p$. If furthermore $H$ is a vector space where $a, b, c$ act as endomorphisms and preserve a subspace $A \subset H$, then $p$ acts as the identity on $H$, whenever $a=i d$ on $A, b=c=0$ on $A$ and $b=i d$ on $H / A$. We apply this to $a=\pi_{20}, b=\pi_{11}, c=\pi_{02}$ and $H=H_{\mathrm{tr}}^{2}(Y, \mathbb{Q}), A=\operatorname{Im}\left(f^{*} H^{2}(S, \mathbb{Q})\right)$.

\section{Proof of theorem 2}

Let $X$ be a smooth projective $n$-fold over a field $k$ of characteristic zero together with a rational point $e \in X(k)$. By [Sch, 1.17] we may again assume that $k$ is algebraically closed. First we need two lemmas about blow-ups on $X$.

Lemma 2. Let $\varphi: Y \rightarrow X$ be a consecutive blow up of $X$ along several smooth centers $W_{j}$ of dimension $\leq 1$ and $q \in C H^{n}(Y \times Y)$ be a projector which is supported in $H \times Y$ for some effective irreducible divisor $H \subset Y$ and acts trivially or as the identity on $H^{2 n-2}(Y, \mathbb{Q})$. Then $p:=(\varphi \times \varphi)_{*} q \in C H^{n}(X \times X)$ is a projector and for all $\alpha \in C H^{*}(X)$ on has $p(\alpha)=\varphi_{*} q\left(\varphi^{*}(\alpha)\right)$. Moreover if one has two orthogonal projectors $q_{1}, q_{2}$ on $Y$ satisfying the same properties, then the corresponding projectors $p_{1}$ and $p_{2}$ are again orthogonal.

Proof. By induction on the number of blow-ups we may assume that there is just one blow-up along a smooth subvariety $W \subset X$. Let $E \subset Y$ be the exceptional divisor and $T \subset \varphi(H) \times X$ the algebraic subset $T:=\varphi(H) \times W \cup(\varphi(H) \cap W) \times X$. 
We have to show that the cycle $\mathfrak{C}:=p \circ p-p \in C H_{n}(\varphi(H) \times X)$ is trivial. Consider the localization sequence

$$
C H_{n}(T) \stackrel{j_{*}}{\rightarrow} C H_{n}(\varphi(H) \times X) \rightarrow C H_{n}(U) \rightarrow 0
$$

where $U:=\varphi(H) \times X \backslash T$. Since $q$ is a projector, the image of $\mathfrak{C}$ in $C H_{n}(U)$ vanishes. Therefore $\mathfrak{C}=j_{*} Q$. If $\operatorname{dim}(W)=0$, we have $Q=0$ by dimension reasons. If $\operatorname{dim}(W)=1$, one gets that

$$
Q=a(\varphi(H) \times W)+b((\varphi(H) \cap W) \times X)
$$

with $a, b \in \mathbb{Z}$.

But for every cohomology class $\alpha \in H^{2 n-2}(X, \mathbb{Q}), p(\alpha)=\varphi_{*} q\left(\varphi^{*}(\alpha)\right)$. If $q$ is zero or the identity on $H^{2 n-2}(X, \mathbb{Q})$, this implies that $p(\alpha)$ is zero or equal to $\alpha$. In both cases $\mathfrak{C}(\alpha)=0$ for every $\alpha \in H^{2 n-2}(X, \mathbb{Q})$ or $\alpha \in H^{0}(X, \mathbb{Q})$. Therefore $a=b=0$ and $p$ is a projector.

If $q_{1}, q_{2}$ are two orthogonal projectors, then one concludes in a similar way that $p_{1} \circ p_{2}$ is supported on $T$ and therefore it vanishes by the same argument as above.

Let $\pi_{i}(S)$ be a special Chow-Künneth decomposition for $S$. Keeping the notation $\pi_{i j}(Y)=\pi_{i j}$ of the previous section, we have a projector

$$
\pi_{2}:=\pi_{20}+\pi_{02}+\pi_{11}-\pi_{20} \pi_{02}-\pi_{11} \pi_{02}-\pi_{20} \pi_{11}+\pi_{20} \pi_{11} \pi_{02}
$$

on $Y$ by propositions 1 and 2 .

Now we choose a Zariski open subset $U \subset Y$ such that $f: U \rightarrow f(U)$ is contained in the smooth part of $f$. Let $T:=Y \backslash U \stackrel{\iota}{\hookrightarrow} Y$ be the complement. From now on we also will assume that (after further blow-ups of $Y$ ) we are in the situation where $T=\bigcup T_{i}$ is a divisor with strict normal crossings. Additionally we choose $U$ small enough so that $f: Z \cap U \rightarrow f(U)$ is étale.

The Leray spectral sequence for $f$ on $U$

$$
H^{k}\left(f(U), R^{\ell} f_{*} \mathbb{C}\right) \Longrightarrow H^{k+\ell}(U, \mathbb{C})
$$

degenerates at $E_{2}$ and each term has a mixed Hodge structure by [Sa] whose lowest weight piece $W_{0} H^{k}\left(f(U), R^{\ell} f_{*} \mathbb{C}\right)$ converges to

$$
W_{0} H^{k+\ell}(U, \mathbb{C})=\operatorname{Im}\left(H^{k+\ell}(Y, \mathbb{C}) \rightarrow H^{k+\ell}(U, \mathbb{C})\right) .
$$

Lemma 3. The projectors $\left.\pi_{i j}\right|_{U \times U}$ act trivially on $W_{0} H^{k}\left(f(U), R^{\ell} f_{*} \mathbb{C}\right)$ if $i \neq k$ or $j \neq \ell$. In particular by the Leray spectral sequence $\pi_{11}$ acts trivially on $W_{0} H^{m}(U, \mathbb{C})$ if $m \neq 2$.

Proof. Let

$$
\mathfrak{D}_{U}^{m}=\bigoplus_{r+s=m} \mathfrak{D}_{U}^{r, s}
$$

be the sheaf of complex valued $m$-currents on $U$. The sequences

$$
0 \rightarrow f^{*} \mathfrak{D}_{f(U)}^{1} \rightarrow \mathfrak{D}_{U}^{1} \rightarrow \mathfrak{D}_{U / f(U)}^{1} \rightarrow 0
$$

are canonically split due to the assumption that $Z \cap U$ is étale over $f(U)$. Therefore we get a canonical decomposition

$$
\mathfrak{D}_{U}^{m}=\bigoplus_{a+b+c+d=m} f^{*} \mathfrak{D}_{f(U)}^{a, b} \wedge \mathfrak{D}_{U / f(U)}^{c, d}
$$


and any element of $H^{i}\left(f(U), R^{j} f_{*} \mathbb{C}\right)$ may be represented by a sum of currents of the form $\omega=f^{*} \alpha \wedge \beta \in f^{*} \mathfrak{D}_{f(U)}^{a, b} \wedge \mathfrak{D}_{U / f(U)}^{c, d}$ with $a+b=i, c+d=j$.

As long as we only consider cohomology of weight zero, which is the image of the cohomology of the compactification, all direct images are defined and therefore currents behave well under correspondences. Every subvariety $W$ of codimension $r$ has an $(r, r)$-current $\delta_{W}$ associated to it. If $\omega=f^{*} \alpha \wedge \beta \in f^{*} \mathfrak{D}_{f}(U)^{a, b} \wedge \mathfrak{D}_{U / f(U)}^{c, d}$, then $\pi_{i 0}(\omega)=\frac{1}{m} i_{*} h^{*} \pi_{i}(S)\left[\alpha \wedge h_{*}\left(\left.\beta\right|_{Z}\right)\right] .\left.\beta\right|_{Z}=0$ unless $c=d=0$ (Z being finite over $S$ ). This proves that $\pi_{i 0}=0$ on every group $H^{k}\left(f(U), R^{\ell} f_{*} \mathbb{C}\right)$ for $\ell \geq 1$ and acts as $\delta_{i k}$ on $H^{k}\left(f(U), f_{*} \mathbb{C}\right)$, because $h_{*}(1)=m \in \mathfrak{D}_{f(U)}^{0,0}$.

Similarly $\pi_{i 2}(\omega)=h^{*} \pi_{i}(S)\left(\alpha \wedge f_{*} \beta\right)$ (see dAMS, thm. 3.3.]). This is nonzero in cohomology only when $c=d=1$ and $a+b=i$, proving the assertion for $\pi_{i 2}$.

In the last case

$$
\pi_{i 1}(\omega)=p r_{2 *}^{f(U) \times U}\left[(1 \times f)^{*}\left(p r_{1}^{*}(\alpha) \wedge \delta_{\pi_{i}(S)}\right) \wedge(f \times 1)_{*}\left(\delta_{D} \wedge p r_{1}^{*}(\beta)\right)\right]
$$

The last term $(f \times 1)_{*}\left(\delta_{D} \wedge p r_{1}^{*}(\beta)\right)$ is zero unless $c+d=1$ by a type argument, in which case one gets

$$
\pi_{i 1}(\omega)=p r_{2 *}^{f(U) \times U}\left[(1 \times f)^{*}\left(p r_{1}^{*}(\alpha) \wedge \delta_{\pi_{i}(S)}\right)\right] \wedge \gamma=f^{*} \pi_{i}(S)(\alpha) \wedge \gamma
$$

with $\gamma \in \mathfrak{D}_{U / f(U)}^{c, d}$. This is zero if $a+b \neq i$.

Lemma 4. $\pi_{2}$ operates trivially on $H^{3}(Y, \mathbb{C})$.

Proof. Take $\beta \in H^{3}(Y, \mathbb{Q})$ and check that $\pi_{20}(\beta)=\pi_{02}(\beta)=0$ by using the formulas $\pi_{j 0}(\beta)=\frac{1}{m} f^{*} \pi_{j}(S)\left(h_{*}(\beta \cap Z)\right)$ and $\pi_{j 2}(\beta)=\frac{1}{m} i_{*} h^{*} \pi_{j}(S)\left(f_{*} \beta\right)$ and the fact that $\pi_{i}(S)$ satisfies property (3) of a Chow-Künneth decomposition.

It remains to show that $\pi_{11}(\beta)=0$. Look at the long exact sequence for cohomology with supports (with $T, U$ as defined above):

$$
\ldots \rightarrow H^{2}(U, \mathbb{C}) \rightarrow H_{T}^{3}(Y, \mathbb{C}) \stackrel{\iota^{*}}{\rightarrow} H^{3}(Y, \mathbb{C}) \stackrel{j^{*}}{\rightarrow} H^{3}(U, \mathbb{C}) \rightarrow \ldots
$$

By the purity of the Hodge structure $H^{3}(Y, \mathbb{C})$, the image of $\iota_{*}$ is generated by the image of $\bigoplus H^{1}\left(T_{i}, \mathbb{C}\right)$ under the sum of the Gysin maps.

Lemma 4 follows therefore from two assertions:

$(1) \pi_{11}(\beta)$ is an element of $\operatorname{Im}\left(H_{T}^{3}(Y, \mathbb{C}) \stackrel{\iota_{*}}{\rightarrow} H^{3}(Y, \mathbb{C})\right)$.

(2) $\pi_{11}$ acts trivially on the image of every $H^{1}\left(T_{i}, \mathbb{C}\right)$ in $H^{3}(Y, \mathbb{C})$.

Ad (1): By Chow's moving lemma, we may assume that $\pi_{11}$ intersects $T \times Y$ properly. This implies that $j^{*} \pi_{11}(\beta)=\left(\left.\pi_{11}\right|_{U \times U}\right)\left(j^{*}(\beta)\right)$ which vanishes by lemma 3. Therefore $\pi_{11}(\beta) \in \operatorname{Im}\left(H_{T}^{3}(Y, \mathbb{C}) \stackrel{\iota_{*}^{*}}{\rightarrow} H^{3}(Y, \mathbb{C})\right)$.

Ad (2): It is even enough to show that $\pi_{11}$ is zero on the image of all maps

$$
\operatorname{Pic}^{0}\left(T_{i} \stackrel{\stackrel{\iota *}{\rightarrow}}{\rightarrow} J^{2}(Y)\right.
$$

to Griffiths' intermediate Jacobian. Namely if we know that

$$
\tau=\pi_{11}: J^{2}(Y) \rightarrow J^{2}(Y)
$$

is the zero map, then the continuous lifting

$$
\bar{\tau}: H^{3}(Y, \mathbb{C}) / F^{2} \rightarrow H^{3}(Y, \mathbb{C}) / F^{2}
$$

also has to vanish. By using complex conjugation this in turn implies that the resulting map on $H^{3}(Y)$ is trivial. 
Now consider a class $\beta \in P i c^{0}(T)$ (without loss of generality we may assume that $T$ is irreducible). Then, using the projector $\pi_{1}(S)=(C \times S) \cap D_{S}$ of $\mathrm{Mu} 2$, we obtain

$$
\begin{gathered}
\pi_{11}\left(\iota_{*} \beta\right)=p r_{2 *}\left[(f \times f)^{*}\left(C \times S \cap D_{S}\right) \cap D \cap\left(\iota_{*} \beta \times Y\right)\right] \\
=p r_{2 *}^{T \times Y}\left[(g \times f)^{*}\left(C \times S \cap V \times S \cap D_{S}\right) \cap(\iota \times 1)^{*}(D) \cap(\beta \times Y)\right]
\end{gathered}
$$

Here $V:=f(T) \stackrel{j}{\hookrightarrow} S$ sits in the commutative diagram

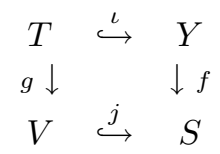

$C$ can be chosen to intersect $V$ properly in a finite set, so

$$
\pi_{11}\left(\iota_{*} \beta\right)=p r_{2 *}^{T \times Y}\left[\left(\sum_{Q \in C \cap V} g^{*} Q \times f^{*} W(Q)\right) \cap(\iota \times 1)^{*}(D) \cap(\beta \times Y)\right]
$$

where $W(Q) \in P i c^{0}(S)$ and hence by suitable rewriting

$$
\pi_{11}\left(\iota_{*} \beta\right)=\sum_{P \in g^{*}(C \cap V) \cap|\beta|} \operatorname{pr}_{2 *}\left[a_{P} P \times(W(P) \cdot D(P))\right]
$$

for some classes $W(P), D(P) \in P i c^{0}(Y)$ and $a_{P} \in \mathbb{Z}$. The lemma follows then from the fact that the cup product map

$$
\operatorname{Pic}^{0}(Y) \times \operatorname{Pic}^{0}(Y) \rightarrow J^{2}(Y)
$$

in Deligne cohomology is zero by $[\mathrm{EV}]$.

Remark. The above argument can also be applied to the projector $\pi_{1}(S)$ as defined in [Sch], since one only needs to add a term of the form $\frac{1}{2} \pi_{3} \circ \pi_{1}$, with the one of $\mathrm{Mu} 2$, therefore a similar argument shows that this new term also acts as zero.

Lemma 5. Assume that $f: Y \rightarrow S$ decomposes $H_{t r}^{2}(Y)$. Then for a suitable choice of curves $\left.\ell_{i} \subset Y(1 \leq i \leq r=\operatorname{rkN} S(Y) \otimes \mathbb{Q})\right)$ and a matrix $B=\left(b_{i j}\right) \in$ $\operatorname{Mat}(r \times r, \mathbb{Q})$, the modified projector

$$
p_{2}(Y)=\pi_{2}(Y)+\sum b_{i, j}\left(\ell_{i} \times T_{j}\right)-\sum b_{i, j}\left(\ell_{i} \times T_{j}\right) \circ \pi_{2}(Y)
$$

acts as the identity on $H^{2}(Y, \mathbb{Q})$.

Proof. By proposition 2 we know that $\pi_{2}(Y)$ acts as the identity on $H_{\mathrm{tr}}^{2}(Y, \mathbb{Q})$. Let $A \in \operatorname{Mat}(r \times r, \mathbb{Q})$ be the matrix describing the action of $\pi_{2}(Y)$ on $N S(Y) \otimes \mathbb{Q}$. By the Hodge conjecture on $Y$, there exist suitable curves $\ell_{i}$ whose cohomology classes form a Poincaré dual vector space to the image of $N S(Y) \otimes \mathbb{Q}$. Let $M \in \operatorname{Mat}(r \times r, \mathbb{Q})$ be the invertible intersection matrix $M=\left(m_{i, j}\right):=\left(\ell_{1}, \ldots, \ell_{r}\right)^{T}\left(T_{1}, \ldots, T_{r}\right)$ and define $B$ to be $M^{-1}(\mathbf{1}-A)$. Now the rest of the proof proceeds as in dAMS: $p_{2}(Y)$ acts via the matrix $M B+A+B A$ on $N S(Y) \otimes \mathbb{Q}$. Now $\pi_{2}^{2}=\pi_{2}$ and we get $A^{2}=A$ and therefore $B A=0$. By definition of $B$, we obtain that $M B+$ $A+B A=M\left(M^{-1}(\mathbf{1}-A)\right)+A=1$. To show that $p_{2}$ is a projector, let us write $p_{2}=\pi_{2}+\beta-\beta \pi_{2}$. Note that $\beta \beta=\beta$, since $B M B=B$. From $B A=0$ we deduce that $\pi_{2} \beta=0$. Therefore $p_{2} \circ p_{2}=\pi_{2}^{2}+\beta^{2}+\beta \pi_{2} \beta \pi_{2}+\pi_{2} \beta-\pi_{2} \beta \pi_{2}+\beta \pi_{2}-\beta \beta \pi_{2}-$ $\beta \pi_{2} \pi_{2}-\beta \pi_{2} \beta=\pi_{2}+\beta-\beta \pi_{2}=p_{2}$ is a projector.

As a corollary we obtain 
Theorem 2. Let $X$ be a smooth projective 3-fold over a field of characteristic 0 such that a blow-up $Y$ of $X$ has a fibration $f: Y \rightarrow S$ which decomposes $H_{t r}^{2}(Y)$. Then $X$ admits a special Chow-Künneth decomposition.

Proof. Choose a blow-up $\varphi: Y \rightarrow X$ such that $Y$ is a smooth projective 3 -fold with an $H^{2}$-decomposing fibration $f: Y \rightarrow S$ of relative dimension one. $S$ admits a special Chow-Künneth decomposition by Mu1. We may also assume that there exists a divisor $Z \subset Y$ which is finite over $S$. By extending the ground field $k$ we may assume that $Z$ and $f$ are defined over $k$ and use [Sch, 1.17.] to reduce back to the smaller field by taking the norm.

Now take the orthogonal set of projectors $p_{0}, p_{1}, p_{5}, p_{6}$ as defined in [Mu2] or Sch], $p_{2}$ as in lemma 5 and $p_{4}=p_{2}^{\text {tr }}$ on $Y$. Apply the non-commutative GramSchmidt process (see proof of proposition 2) by modifying only $p_{2}$ and $p_{4}$. By lemmas 4 and 5 the new projectors, again denoted by $p_{0}, p_{1}, p_{2}, p_{4}, p_{5}, p_{6}$ have properties (1) and (3) of a Chow-Künneth decomposition for $Y$, since the orthogonality guarantees the vanishing of $p_{i}$ on $H^{j}(Y)$ for $i \neq 3, j$. Finally we define

$$
p_{3}:=\Delta_{Y}-\sum_{i \neq 3} p_{i}
$$

Then the set $\left\{p_{i}\right\}$ satisfies properties (1)-(3) of a special Chow-Künneth decomposition for $X$.

Final remarks. It is easy to see that parts of properties (4)-(6) are satisfied for our choice of projectors. But (5) and (6) for $C H^{2}(X)$ remain open.

\section{REFERENCES}

[B] A.Beauville: Sur l'anneau de Chow d'une variété abélienne, Math. Ann. 273, 647-651 (1986). MR 87g:14049

[dAMS] P.L.del Angel, S.Müller-Stach: Motives of uniruled 3-folds, Compositio Math. 112 (1998), 116. MR 99c:14004 MR

[dBR] S.del Baño Rollin: On the motive of some moduli spaces, preprint, 1996.

[dJ] A.J.de Jong: Smoothness, semistability and alterations, Inst. Hautes Études Sci. Publ. Math., 1996, 51-93. MR 98e:14011

[D] P.Deligne: Théorie de Hodge II, Inst. Hautes Itudes Sci. Publ. Math. (1971), 5-57. MR 58:16653a

[DM] C.Deninger, J.Murre: Motivic decomposition of abelian schemes and the Fourier transform, J. Reine Angew. Math. 422, 201-219 (1991). MR 92m:14055

[EV] H.Esnault, E.Viehweg: Deligne Beilinson cohomology, Beilinson's conjectures on special values of L-functions, Academic Press Perspectives 4, 43-92 (1988). MR 89k:14008

[Fu] W.Fulton: Intersection theory, Grundlehren, Springer, New York, (1984). MR 85k:14004

[GM] B.Gordon, J.Murre: Chow groups of elliptic modular varieties, preprint (1996).

[J] U.Jannsen: Motivic sheaves and filtrations on Chow groups, Motives, AMS Proc. of Symp. 55, 245-302 (1994). MR 95c:14006

[K] S.Kleiman: Motives, Proc. ICM Oslo 1970, Noordhoff, 53-82 (1970). MR 52:3152

[Kö] B.Köck: Chow motif and higher Chow theory of $G / P$, Manuscripta Mathematica 70, 363-372 (1991). MR 91m:14077

[Kü] K.Künnemann: On the Chow motive of an abelian scheme, Motives, Seattle, AMS Proc. of Symp. 55, 189-205 (1994). MR 95d:14009

[M] Y.Manin: Correspondences, motives and monoidal transforms, Mat. USSR Sbornik 6, 439-470 (1968).

[Mum] D.Mumford: Abelian Varieties, Oxford University Press, (1970). MR 44:219

[Mu1] J.Murre: On a conjectural filtration on the Chow groups of an algebraic variety $\mathbf{I}$ and II, Indag. Math. 4, 177-188 and 189-201 (1993). MR 94j:14006a 
[Mu2] J.Murre: On the motive of an algebraic surface, J. Reine Angew. Math. 409, 190-204 (1990). MR 91g:14003

[Sa] M. Saito: Modules de Hodge polarisables, Publ. RIMS 24, 849-995 (1988). MR 90k:32038

[S] S.Saito: Motives and filtrations on Chow groups, Invent. Math 125, 149-196 (1996). MR 97i:14002

[Sch] A.Scholl: Classical motives, in Motives, AMS Proc. of Symp. 55, 163-187 (1994). MR 95b:11060

[Sh] A.Shermenev: The motive of an abelian variety, Functional Analysis 8, 55-61 (1974).

Departamento de Matemáticas, Uam i, Mexico City, Mexico

Current address: Fachbereich 6, University Essen, 45117, Essen, Germany

E-mail address: pedro.del.angel@uni-essen.de

Fachbereich 6, University Essen, 45117 Essen, Germany

E-mail address: mueller-stach@uni-essen.de 